\title{
Real time data collection and processing using mobile technology: A public health perspective
}

\author{
Dr. N. Siriwardena \\ Postgraduate Institute of medicine, University of Colombo, Sri Lanka. \\ Dr. M. Dharmawardhana \\ Postgraduate Institute of medicine, University of Colombo, Sri Lanka. \\ Contact e-mail address: siriwardenanishan@ gmail.com \\ eHealth Sri Lanka 2010,1(suppl.1):S7 \\ DOI: http://dx.doi.org/10.4038/sljbmi.v1i0.3539
}

Only the Abstract is available

\begin{abstract}
Health information system in preventive healthcare domain in Sri Lanka is built on a solid foundation laid by a vast number of public healthcare workers since its inception. Information flow from field health care personnel to the specialists and policy makers in the center is managed manually by using standard formats. A considerable portion of working time of the primary healthcare personnel is spent on the preparation of data sheets and forwarding it to the next level of information flow. Accuracy and timeliness of data is affected by varied factors within the system and outside. Accesses to data are hindered by inherent qualities of manual information systems. Therefore, integration of information technology with the existing paper based system could enhance the quality of the data and reduce the time spent on preparation of data sheets by heathcare personnel. Taking this into consideration a system is designed to show the capabilities of mobile technology to collect and record data and process and update it to real time. The system elaborates the ability to collect data, process initial validation on site and update it real time to the local server with feed back, using mobile broadband technology. In any case where the mobile broadband is not available, the system has the ability to buffer the entered data and update the database when the device goes online. If the device fails to go online for a certain time, it will be required to be taken to the local data server and connect, where the updating will occur instantaneously. The data then will be processed and sent to the upper level of the information flow hierarchy.
\end{abstract}

Keywords - preventive health care, field health care, Accuracy and timeliness of data 